\title{
Simulation of the Space Charge Effect in RHIC *
}

\author{
G.F. Dell and S. Peggs \\ Brookhaven National Laboratory \\ Upton, New York 11973, USA
}

\begin{abstract}
Space charge forces, representing the weak-strong case, are simulated by kicks from a line charge having a 2D-Gaussian transverse charge distribution. A series of particles having initial coordinates consistent with the dimensions of the injected beam are tracked sequentially, and tunes are obtained from analysis of the coordinates, $x, x^{\prime}, y$, and $y^{\prime}$, at the end of each turn. Stability is tested using $30 \mathrm{~K}$ turn tracking runs during which the momentum error $\delta$ varies as $\delta=\hat{\delta} \sin \left(2 \pi Q_{\mathrm{s}} t\right)$.
\end{abstract}

\section{INTRODUCTION}

RHIC will have 57 bunches of $10^{9} \mathrm{Au}$ ions with a spacing of $67.3 \mathrm{~m}$. There is a $9.8 \mathrm{~m}$ region on either side of the interaction point in which the colliding beams will be collinear. They will then be separated by the DX dipoles. With this geometry there are no parasitic beam-beam collisions. Hence the tune shift from beam-beam forces is limited to head on beam-beam collisions at the interaction points and is small.

The tune shift from the space charge is expected to be $\sim$ 0.021. As RHIC will operate between the 5th and 6th integer resonances at nominal tunes of $\nu_{\mathrm{x}}=28.190$ and $\nu_{\mathrm{x}}=29.180$, the anticipated space charge tune shift may drive particles on the edge of the beam to the 5th integer resonances at 28.200 and 29.200. The present study was made to explore possible limitations to the dynamic aperture caused by space charge forces.

The beam has transverse dimensions much smaller than the bunch length and is treated as a line charge having a transverse charge distribution represented by a 2-dimensional Gaussian. Test particles at an arbitrary displacement from the axis of the beam experience kicks attributed to the space charge force. The tunes of small amplitude betatron motion are depressed by as much as 0.021 , and the arc quadrupoles are adjusted to restore the tunes to the nominal values of $28.190,29.180$. However, particles with large amplitudes experience little space charge force and have tunes that can approach or cross the 5th integer resonances at $\nu_{\mathrm{x}}=28.200$ and $\nu_{\mathrm{y}}=29.200$. Information on tune and amplitude growth of the motion is obtained by tracking on a grid with initial displacements de®ned by the action $J_{\mathrm{t}}=(n \sigma)^{2} / 2 \beta=J_{x}+J_{y}$ with $0.5 \leq n \leq 7$.

\section{THEORY}

The electric $\circledR$ eld from a 2D-Gaussian charge distribution at a point $(\mathrm{x}, \mathrm{y})$ has been expressed in terms of the complex error function by Bassetti and Erskine [1]

\footnotetext{
*Work performed under the auspices of the U.S. Department of Energy
}

$$
\begin{aligned}
& E_{\mathrm{x}}=\frac{Q}{2 \epsilon_{0} \sqrt{\pi S_{\mathrm{xy}}}} \operatorname{Im}\left[W\left(\frac{z}{\sqrt{S_{\mathrm{xy}}}}\right)-e^{-\left(\frac{x^{2}}{2 \sigma_{\mathrm{x}}^{2}}+\frac{y^{2}}{2 \sigma_{\mathrm{y}}^{2}}\right)} W\left(\frac{z_{1}}{\sqrt{S_{\mathrm{xy}}}}\right)\right] \\
& E_{\mathrm{y}}=\frac{Q}{2 \epsilon_{0} \sqrt{\pi S_{\mathrm{xy}}}} \operatorname{Re}\left[W\left(\frac{z}{\sqrt{S_{\mathrm{xy}}}}\right)-e^{-\left(\frac{x^{2}}{2 \sigma_{\mathrm{x}}^{2}}+\frac{y^{2}}{2 \sigma_{\mathrm{y}}^{2}}\right)} W\left(\frac{z_{1}}{\sqrt{S_{\mathrm{xy}}}}\right)\right]
\end{aligned}
$$

The above expressions are valid when $\sigma_{\mathrm{x}}>\sigma_{\mathrm{y}}$, where:

$$
\begin{gathered}
S_{\mathrm{xy}}=2\left(\sigma_{\mathrm{x}}^{2}-\sigma_{\mathrm{y}}^{2}\right), \\
z=x+i y, \\
z_{1}=x \frac{\sigma_{\mathrm{y}}}{\sigma_{\mathrm{x}}}+i y \frac{\sigma_{\mathrm{x}}}{\sigma_{\mathrm{y}}}
\end{gathered}
$$

and

$$
W(z)=e^{-z^{2}}\left[1+\frac{2 i}{\sqrt{\pi}} \int_{0}^{z} e^{u^{2}} d u\right]
$$

is the complex error function.

Okamoto and Talman [2] have used Pade approximations for rapid evaluation of the complex error function. This formalism has been incorporated in Teapot [3] to evaluate the beam-beam force. The calculation of the kick is divided into three cases according to the proßle of the beam cross section: 1). when $\sigma_{\mathrm{X}}$ and $\sigma_{\mathrm{y}}$ are within one part in $10^{4}$ of being equal, a radial Gaussian dependence, $\left(1-e^{-r^{2} / 2 \sigma^{2}}\right)$ is used, 2). when $\sigma_{\mathrm{x}}>\sigma_{\mathrm{y}}$, Eqn.1 - Eqn.6 are used, and 3). when $\sigma_{\mathrm{y}}>\sigma_{\mathrm{x}}$, x and y are interchanged in Eqn.1 - Eqn.6. Elements of class ${ }^{\text {abeambeam }}{ }^{\circ}$, one of the Teapot elements with ${ }^{\text {a }}$ extended allowed parameters ${ }^{\circ}$, are used to generate kicks from the charge distribution. These elements are intended for calculation of the beam-beam force and are usually placed at the interaction points.

In the present study we use 144 of these elements to simulate the space charge force and have distributed them smoothly at the $\mathrm{SF}$ and SD sextupoles in all six arcs. The different optical functions at SF, where $\sigma_{\mathrm{x}}>\sigma_{\mathrm{y}}$, and $\mathrm{SD}$, where $\sigma_{\mathrm{y}}>\sigma_{\mathrm{x}}$, require two families, and the number of particles/bunch as well as the local values of $\sigma_{\mathrm{x}}$ and $\sigma_{\mathrm{y}}$ must be speci®ed for each.

Keil [4] considers the beam-beam force and points out the electric and magnetic components differ by a factor of $\beta^{2}$. The magnitude of the kick given to the test particle depends upon whether the test particle is travelling with, or against, the beam. For the head on beam-beam effect the test particle travels against the beam, and the electric and magnetic $®$ elds add as $\left(1+\beta^{2}\right)$. For space charge the test particle travels with the beam, and the 
electric and magnetic $® e l d s$ subtract as $\left(1-\beta^{2}\right)$ and contribute a $1 / \gamma^{2}$ factor to the space charge force.

We make use of the transverse dependence of the two dimensional charge distribution given by the beam-beam formalism in Teapot. Compensation for the difference between tune shifts from beam-beam and space charge forces can be made by adjusting the number of particles per bunch until the observed tune shifts equal the Laslett tune shift, Eqn.7. However, for the present study where kicks are present at all SF and SD sextupoles, the observed tune shifts are suf®ciently close to the Laslett tune shift that no change of $N_{\mathrm{b}}$ is necessary.

$$
\Delta \nu=-\frac{N_{\mathrm{b}} r_{\mathrm{p}} R_{0} Z^{2}}{2 \sqrt{2 \pi} \beta^{2} \gamma^{3} \epsilon_{\mathrm{rms}} \sigma_{\mathrm{L}} A}
$$

Where $N_{\mathrm{b}}=10^{9}$ is the number of gold ions per bunch, $r_{\mathrm{p}}=$ $1.510^{-18} \mathrm{~m}$ is the classical proton radius, $R_{0}=610.18 \mathrm{~m}$ is the average radius of RHIC, $\gamma=12.89$ at injection, $\epsilon_{\mathrm{rms}}=$ $\sigma^{2} / \beta=1.29710^{-7} m, \sigma_{\mathrm{L}}=0.9961 \mathrm{~m}$, is the $r m s$ bunchlength, and $\mathrm{Z}=79, \mathrm{~A}=197$ are the charge and mass number. For fully stripped gold, $\Delta \nu=-0.021$.

\section{ERRORS}

\section{A. Position}

Errors assigned to dipole position are $\sigma_{\mathrm{x}}=\sigma_{\mathrm{y}}=0.5 \mathrm{~mm}$ and to dipole orientation are $\sigma_{\theta}=1$ mradian. Determination of the center and orientation of the ${ }^{\circledR e l d s}$ in quadrupoles using colloidal cells[5] justi®es using $\sigma_{\mathrm{x}}=\sigma_{\mathrm{y}}=0.25 \mathrm{~mm}$ and $\sigma_{\theta}=0.5 \mathrm{mra}-$ dian for the position and orientation errors of all quadrupoles.

\section{B. Magnetic}

Magnetic harmonic errors[6], $a_{\mathrm{n}}$ and $b_{\mathrm{n}}$ with $1 \leq n \leq 9$, were obtained from magnetic measurements on 34 arc dipoles and 36 arc quadrupoles. Harmonic errors for special insertion magnets, D0 $(10 \mathrm{~cm})$ and DX $(18 \mathrm{~cm})$ dipoles and the $13 \mathrm{~cm}$ triplet quadrupoles are based on a combination of magnet calculations and measurements of test magnets.

\section{SIMULATION}

Tracking at constant momentum errors of $\delta=0$ and $\pm 0.1\left(\% \pm 2.5 \sigma_{\mathrm{p}} / p\right)$ is performed for test particles with initial displacements de®ned by $J_{\mathrm{t}}=J_{\mathrm{x}}+J_{\mathrm{x}}=(n \sigma)^{2} / 2 \beta$ for $0.5 \leq$ $n \leq 7$ with values of the initial $x$ and $y$ satisfying $J_{\mathrm{x}} / J_{\mathrm{t}}=$ $0.96,0.75,0.50,0.25$ and 0.04 . Each particle is tracked for 512 turns, and the values of $x, x^{\prime}, y$, and $y^{\prime}$ relative to the closed orbit are written to a ßle for post processing from which the tunes at each amplitude are obtained.

Stability is determined using $30 \mathrm{~K}$ turn tracking runs during which the momentum error $\delta$ varies as $\delta=\hat{\delta} \sin \left(2 \pi Q_{\mathrm{s}} t\right)$. As a line charge is assumed, there is no longitudinal variation of the charge density in a bunch.

Four different seeds are used to initiate the generation of random displacements, rotations, and magnet ®eld errors. In each case two families of skew quadrupoles are used to globally decouple the betatron motion, and the arc quadrupoles are adjusted to obtain the desired tunes.

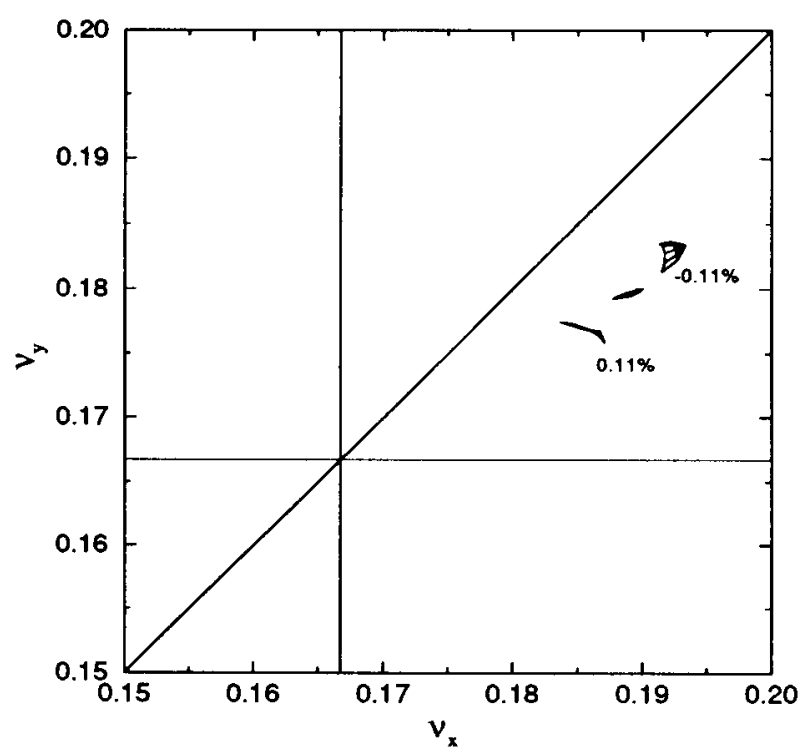

Figure. 1. Dependence of tune on $\delta, J_{\mathrm{t}}$, and aspect ratio $x / y$ with all magnetic ®eld and random positioning and orientation errors included. The nominal tunes are $\nu_{\mathrm{x}}=28.190, \nu_{\mathrm{y}}=$ 29.180 , and at injection, below transition, the chromaticities $\xi_{\mathrm{x}}=\xi_{\mathrm{y}}=-3$.

\section{RESULTS}

Tunes are obtained by averaging the phase advance per turn in each plane, and the results are plotted in tune space with one set of lines connecting particles with equal action and another set of lines connecting particles having the same ratio of $x / y$. Test particles were located wherever two or more lines intersect. ${ }^{a}$ Leafprints ${ }^{\circ}$ showing the dependence of $\nu_{\mathrm{x}}$ and $\nu_{\mathrm{y}}$ on amplitude and aspect ratio $x / y$ are shown in Figure 1 when only magnetic, positioning, and orientation errors are present. In general the tunes decrease as the amplitude increases. The addition of the space charge force depresses the tunes of particles at small amplitudes. The ${ }^{\text {a leafprint }}{ }^{\mathrm{o}}$ in Figure 2 includes the effect of space charge. The arc quadrupoles have been adjusted to restore tunes at small amplitude to the nominal tunes. The leaves for $\delta \neq 0$ are distorted; the tunes at large amplitude include shifts from magnetic ®eld errors, while the tunes at small amplitudes also show a nonlinear chromatic dependence. This feature results from the test particle oscillating around a closed orbit that is displaced by $\eta_{\mathrm{x}} \delta$ from the center of the charge distribution.

Figure 3 shows results of a ${ }^{\circledR}$ ner scan intended to search for tune distortion near the resonances. The test is most sensitive for the $2 \sigma$ and $3 \sigma$ lines and shows no peculiarities.

The stability of particles has also been tested by tracking all particles in the mesh for $30 K$ turns. Figure 4 shows survival plots for four particles representing different error distributions. Solid lines connect data without space charge forces and dashed lines connect data with space charge forces included. The dynamic aperture is consistent with $10 \sigma$ and the plateau shows that all particles having initial amplitudes $\leq 10 \sigma$ survived the requested $30 \mathrm{~K}$ turns. There is no signi ${ }^{\circledR}$ cant decrease of the maximum dynamic aperture when space charge forces are included. As mentioned above, the regions most sensitive to perturbations 


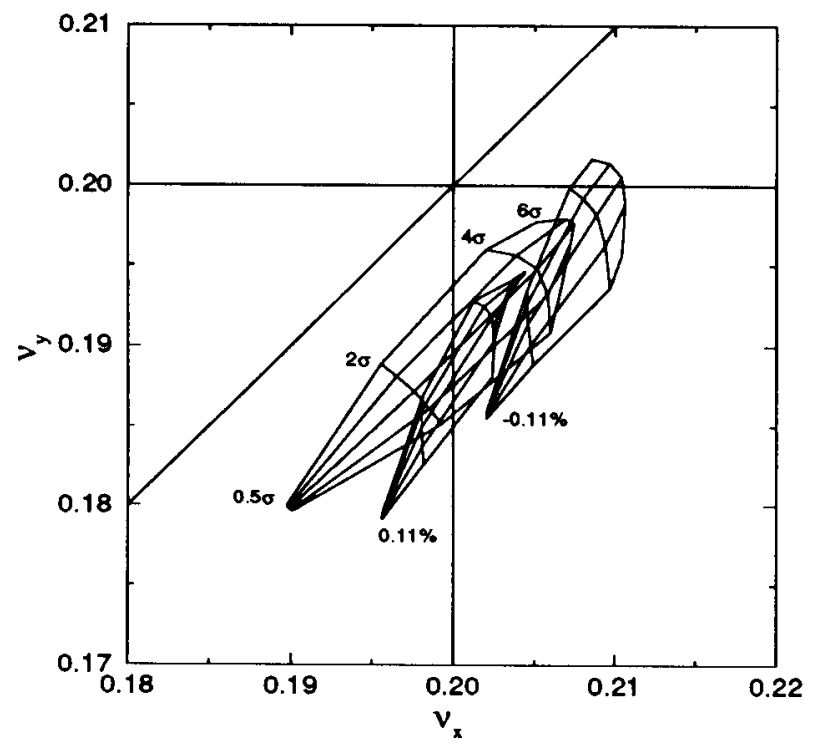

Figure. 2. ${ }^{\circ}$ Leafprint ${ }^{\mathrm{o}}$ when space charge forces are added to the con $®$ guration used in Figure 1. The nominal tunes have been shifted to $\nu_{\mathrm{x}}=28.210, \nu_{\mathrm{y}}=29.200$ to restore the tune at small amplitudes to $\nu_{\mathrm{x}}=28.190, \nu_{\mathrm{y}}=29.180$.

from the 5 th integer resonaces are $2 \leq n \leq 3$ for $\nu_{\mathrm{x}}=28.200$ and $n \geq 4$ for the $\nu_{\mathrm{y}}=29.200$ resonance. The plateau in Figure 4 shows that all particles having initial amplitudes in these ranges survived for the requested $30 \mathrm{~K}$ turns and that, at the level of $30 \mathrm{~K}$ turns, their stability is not limited by the 5 th integer resonances.

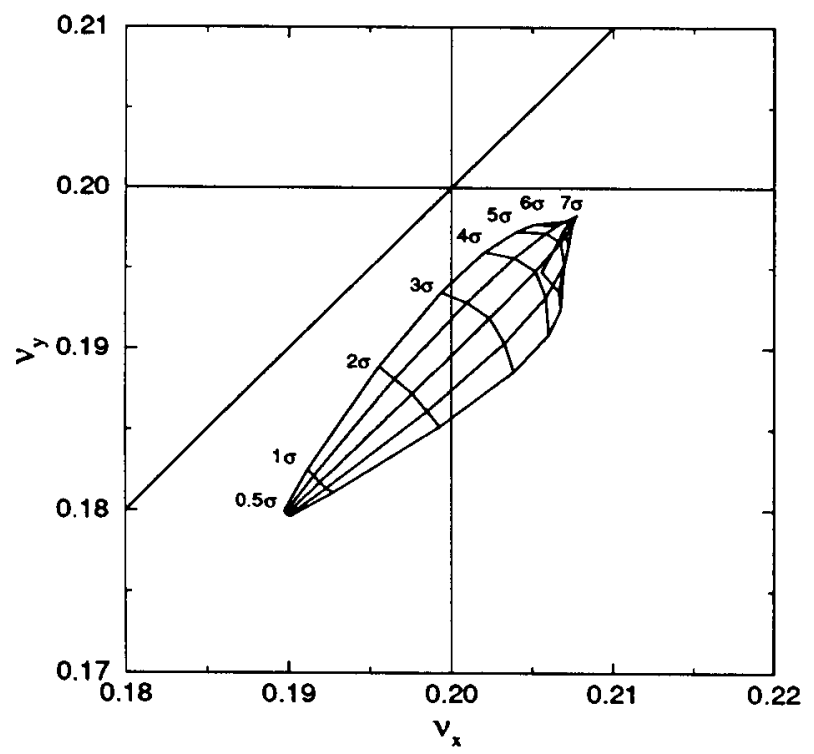

Figure. 3. ${ }^{\circ}$ Leafprint ${ }^{\mathrm{o}}$ at $\delta=0.0 \%$ using a ${ }^{\circledR}$ ner mesh to explore the 5 th integer resonances.

\section{References}

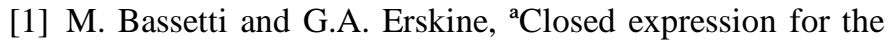
electrical ®eld of a two-dimensional Gaussian charge ${ }^{\mathbf{o}}$, CERN-ISR-TH/80-06,(Unpublished).

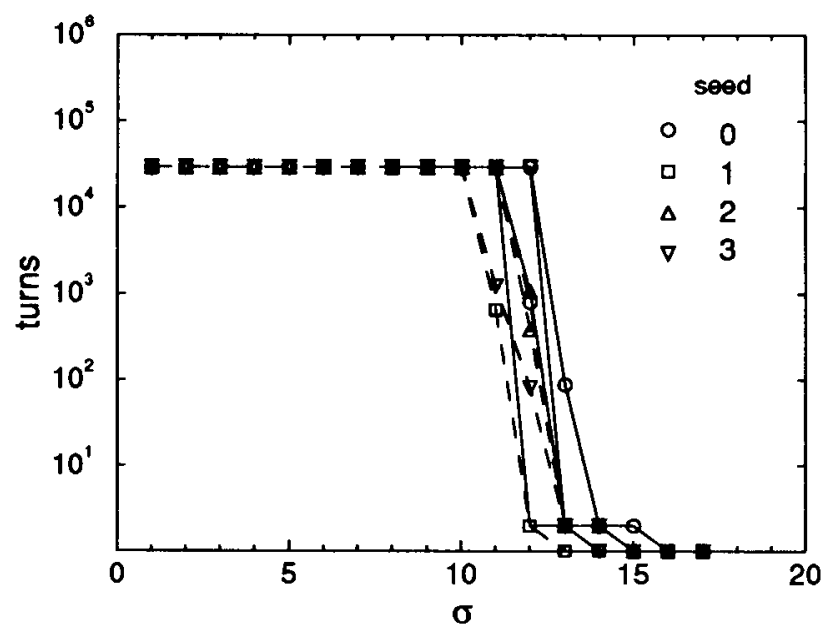

Figure. 4. Comparison of dynamic aperture with(dashed) and without(solid) space charge at $\delta=0.0 \%$.

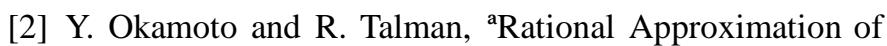
the Complex Error Function and the electric ${ }^{\circledR}$ eld of a Twodimensional Gaussian Charge Distribution', Report CBN 80-13, Cornell University, Sept. 1980.

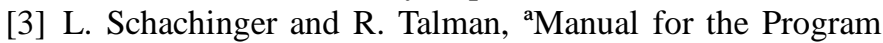
Teapot ${ }^{\circ}$, Cornell Univ., (November 20,1994).

[4] E. Keil, CERN Accelerator School, Rhodes, Greece, Sept. 20 - Oct.1, 1993, Report CERN SL/94-78 (AP).

[5] D. Trbojevic et al., a Alignment and Survey of the Elements in $\mathrm{RHIC}^{\mathrm{o}}$, these proceedings.

[6] J. Wei et al., ' Field Quality Evaluation of the Superconducting Magnets for the Relativistic Heavy Ion Collider ${ }^{\circ}$, these proceedings. 